\title{
THE NUTRIENT CONTENT OF SELECTED SOUTH AFRICAN LAMB AND MUTTON ORGAN MEATS (OFFAL)
}

Bester, M. *; Schönfeldt, H.C.; Pretorius, B. \& Hall, N.

Department of Animal and Wildlife Science, Institute of Food, Nutrition and Well-being, University of Pretoria, Gauteng, South Africa

*Corresponding author. E-mail addresses: posbusmarina@gmail.com, u28429304@tuks.co.za (M. Bester).

\section{Highlights}

- In correct portions, lamb and mutton organs are nutrient dense animal source foods.

- Selected organ meats were "excellent sources of", and "very high in" iron.

- Selected organ meats were "high in" protein, zinc, iron and phosphorous.

- Organs found to be very high in iron were mutton lungs, mutton spleen and lamb lungs.

- The consumption of organ meats should encouraged in South African dietary guidelines.

\section{Introduction}

Organ meats, also known as edible by-products or organ meats, have been overlooked in the past in dietary guidelines and recommendations, irrespective of their potential contribution to food and nutrition security in South Africa. Limited information is available on the composition of South African organ meats as cooked and consumed at home. This limited information includes a recent study done by Van Heerden \& Morey (2014) investigating the nutrient content of South African $\mathrm{C}^{*}$ beef organ meats. This study confirmed that significant amounts of iron and zinc can be found in some beef organs which compared favourably with beef muscle meat cuts and that beef organ meats can be recommended as a good, low cost, nutritious food product (Van Heerden \& Morey, 2014). Small ruminants (goats and sheep) are an integral part of small holder farming systems in South Africa (Tshabalala, Strydom, Webb, \& De Kock, 2003) and could potentially play a positive role in food and nutrition security in these communities. 
In view of rapid population growth in a disease- and poverty-ridden world, the availability of affordable, nutrient dense animal source foods such as organ meats needs to be investigated closely. Known composition data on these foods will enable better consumption recommendations to be made as part of pro-active approaches in eradicating malnutrition and non-communicable diseases (NCDs). Therefore the potential nutritional contribution of these animals' organ meats should also be determined.

Nutrients of concern and generally lacking in South African diets are vitamin A, iron, zinc and B vitamins (Shisana, et al., 2014). Meat is an important nutrient dense food commodity which contributes to nutrients of concern in the South African diet (McAfee, et al., 2010). Meat is however also one of the most expensive items in the food basket. It is believed that organ meats, often also referred to as "offal" or the "fifth quarter", are affordable, alternative nutrient dense animal source foods. The South African National Food Consumption Survey (NFCS), published in 2005, reported that large amounts of organ meats are consumed by children in lower income households in both urban and rural regions (Labadarios, et al., 2005). However the report did not specify which organs were consumed.

The purpose of this study was to determine the nutrient content of selected lamb and mutton organ meats and to determine the possible contribution to the South African diet. This article reports on the nutritional content of raw and cooked $\mathrm{A} 2 *$ lamb and $\mathrm{C} 2 *$ mutton tongues, intestines, stomachs, spleens, lungs, kidneys and livers and the potential contribution of these products to better, affordable, nutrition in South Africa. Nutrients analysed in this study were Crude Protein, Fat, Calcium, Phosphorus, Magnesium, Iron, Manganese, Zinc, Potassium and Sodium.

*A2 lamb and C2 beef and mutton referred to in this article describes the products' age and fatness as per "The South African Red Meat classification system" (South African Meat Industry Company, 2016). "A" refers to a young animal with no permanent incisors whereas " $C$ " refers to an animal with a full set of teeth. A fatness code of "2" refers to a "lean" animal.

\section{Materials and Methods}

In South Africa, lamb and mutton meat are regarded as two distinctly different products. Although they are derived from same species of animal, significant compositional 
differences have been found by previous studies between sheep of different ages (Sainsbury, Schönfeldt, \& Van Heerden, 2011). The nutrient content of different organ meats from both lamb A2 class carcasses and mutton C2 class carcasses was determined and will be reported separately.

\section{Sample Procurement}

Unlike most commercial lamb and mutton retail cuts, where distinction is made between "lamb" and "mutton" on a retail level, organ meats from these animals are usually just labelled "sheep" offal in store. However many abattoirs in South Africa sell offal directly to surrounding communities. Thus the abattoir is an important point of sale and therefore, for this study, lamb- and mutton organ meat samples were procured directly from two abattoirs in Gauteng, South Africa in the Pretoria and Bronkhorstspruit areas. This was also deemed the best method of sample procurement to ensure that samples were lamb or mutton organ meats according to official abattoir classification, and also with the classifications $\mathrm{A} 2 *$ and $\mathrm{C} 2 *$ respectively. The lamb and mutton organ meats included in this study were hearts, livers, lungs, kidneys, tongues, spleens, stomachs, intestines. Six samples of each lamb- and mutton organ meat were procured based on availability ( $n=8 \times 6)$.

\section{Sample Preparation}

All lamb and mutton organs were washed, scrubbed and cleaned with water to remove all remaining manure and stomach contents, as would be done by the consumer on household level. Three samples, from three different animals, of each organ meat $(n=8 \times 3)$ were selected for raw analysis, placed in airtight bags, labelled, frozen and stored at the University of Pretoria in the freezer of the Department of Animal and Wildlife sciences. The remaining three samples of each organ $(n=8 \times 3)$ were prepared for cooking. Three samples of each of the eight lamb organ meat products, and eight of each of the mutton organ meat products were cooked according to a standardised moist heat cooking method. The samples were cooked and prepared in the experimental kitchen of the Department of Consumer Science at the University of Pretoria. The cooking method used, was developed to simulate the cooking processes used at home by most South Africans. The cooking methods most commonly used were derived from research done with a focus group by Duvenage, 
Schönfeldt \& Vermeulen (2011), amongst the lower income population groups in the Limpopo Province (Duvenage, Schönfeldt, \& Vermeulen, 2011) as well as a consumer survey on perceptions towards red meat in the Gauteng province (Vermeulen, Schönfeldt \& Pretorius, 2014). Stewing and braising were the cooking methods most commonly used to cook meat products in South Africa according to both studies. Stewing and braising involves cooking and serving food in a small amount of liquid and thus retaining more nutrients than food cooked in water. Organ meats naturally contain a significant amount of fluids and fat. At the hand of this information it was decided to cook each organ it its own small disposable aluminium oven pan, covered securely with aluminium foil that it would cook in its own liquids. Each organ meat product was cooked to an internal temperature of $75^{\circ} \mathrm{C}$, which is the internal temperature recommended for human consumption of organ meats (Brown, 2010). The covered foil pans were placed on the middle oven racks of the experimental kitchen's built in AEG Competence ovens using a convection oven setting of $160^{\circ} \mathrm{C}$. These ovens are maintained and calibrated for scientific use. Samples were weighed before and after cooking to obtain cooking data and yield factors. Cooked samples were dissected and weighed as separate edible and inedible fractions. Yield factors were calculated as the percentage of the difference between total raw weight and cooked edible portion weight of each organ.

\section{Nutrient Analysis}

For raw nutritional analysis all cartilage, excessive subcutaneous fat and inedible matter were removed from each sample. Thereafter the raw samples were cubed, ground, placed in airtight freezer bags and frozen. The cooked samples were cooled to room temperature, dissected into fat, cartilage and meat for physical composition data. Edible fractions (meat and fat) were cubed, ground and placed in airtight freezer bags. All nutrient analysis was done at the NutriLab of the University of Pretoria. The details and references for each method of analysis can be found in Table 1.

\section{Moisture Content and Freeze Drying}

Each raw ground sample was thawed and homogenized before moisture analysis was carried out. Moisture content analysis of the cooked samples was done on the same day as cooking and grinding. Moisture content analysis was done in duplicate for both raw and 
cooked samples. All samples were freeze dried to obtain a homogenous sample for the rest of the analyses.

\section{Statistical Analysis}

Data was collected, captured and prepared for statistical analysis in Microsoft Excel. Descriptive statistics were done by a qualified statistician using GenStats software (Windows Genstats, 2000). All data were analysed by analysis of variance.

Table 1: Methods of analysis references (AOAC, 2000)

\begin{tabular}{|c|c|}
\hline \multicolumn{2}{|r|}{ General Analyses } \\
\hline Moisture Determination & $\begin{array}{l}\text { AOAC, 2000. Official method of analysis } 934.01\left(17^{\text {th }} \text { Edition) Volume I. }\right. \\
\text { Association of Official Analytical Chemists, Inc., Maryland, USA }\end{array}$ \\
\hline $\begin{array}{l}\text { Dry Matter Ashing (inorganic } \\
\text { fraction) }\end{array}$ & $\begin{array}{l}\text { AOAC, 2000. Official method of analysis } 932.05\left(17^{\text {th }} \text { Edition) Volume I. }\right. \\
\text { Association of Official Analytical Chemists, Inc., Maryland, USA }\end{array}$ \\
\hline Crude protein (CP) & $\begin{array}{l}\text { AOAC, 2000. Official method of analysis } 968.06\left(17^{\text {th }} \text { Edition) Volume I. }\right. \\
\text { Association of Official Analytical Chemists, Inc., Maryland, USA }\end{array}$ \\
\hline $\begin{array}{l}\text { Ether Extraction (EE) for crude } \\
\text { fat }\end{array}$ & $\begin{array}{l}\text { AOAC, 2000. Official method of analysis } 920.39\left(17^{\text {th }} \text { Edition) Volume I. }\right. \\
\text { Association of Official Analytical Chemists, Inc., Maryland, USA }\end{array}$ \\
\hline \multicolumn{2}{|r|}{ Mineral Analysis } \\
\hline $\begin{array}{l}\text { Sample preparation (Ca, } \mathrm{Mg}, \mathrm{Cu} \text {, } \\
\mathrm{Mn}, \mathrm{K}, \mathrm{Na}, \mathrm{Fe}, \mathrm{Zn})\end{array}$ & $\begin{array}{l}\text { AOAC, 2000. Official method of analysis } 935.13\left(17^{\text {th }} \text { Edition) Volume I. }\right. \\
\text { Association of Official Analytical Chemists, Inc., Maryland, USA }\end{array}$ \\
\hline Sample preparation (P) & $\begin{array}{l}\text { AOAC, 2000. Official method of analysis 968.08.D.b ( } 17^{\text {th }} \text { Edition) Volume I. } \\
\text { Association of Official Analytical Chemists, Inc., Maryland, USA }\end{array}$ \\
\hline $\begin{array}{l}\text { Mineral content }(\mathrm{Ca}, \mathrm{Mg}, \mathrm{Cu}, \mathrm{Mn} \text {, } \\
\mathrm{K}, \mathrm{Na}, \mathrm{Fe}, \mathrm{Zn})\end{array}$ & $\begin{array}{l}\text { Giron, H. C., 1973. Atomic Absorption Newsletter 12, 28. Perkin Elmer } \\
\text { Atomic Spectrophotometer }\end{array}$ \\
\hline Phosphorus (P) & $\begin{array}{l}\text { AOAC, 2000. Official method of analysis } 965.17\left(17^{\text {th }} \text { Edition) Volume I. }\right. \\
\text { Association of Official Analytical Chemists, Inc., Maryland, USA }\end{array}$ \\
\hline
\end{tabular}

\section{Results and Discussion}

\section{Cooking data and yield factors}

Cooking data and yield factors for mutton and lamb organs are presented in Table 2. Raw weights for mutton organs range between $80 \mathrm{~g}$ (kidneys) and $2189 \mathrm{~g}$ (stomachs) and for lamb organs between 51g (kidneys) and 2 009g (intestines). Cooked mutton edible portions 
ranged between $29.1 \mathrm{~g}$ (kidneys) and 1 289g (stomachs). There was no significant difference (in terms of weight in grams) between the edible portions of cooked mutton hearts, kidneys, spleens, lungs and tongues which can be seen as the group of smaller organs from a sheep carcass, yielding between $29.1 \mathrm{~g}$ (kidneys) and 318g (lungs). The larger organs, namely intestines, livers and stomachs, had edible portion yields between $477 \mathrm{~g}$ (livers) and $1289 \mathrm{~g}$ (stomachs) and did not differ significantly from each other but did differ significantly from the smaller organs (hearts, kidneys, spleens, lungs and tongues).

As was found in a study done in New Zealand on lamb organs (Purchas \& Wilkinson, 2013), it was difficult to distinguish between subcutaneous fat, intermuscular fat and muscle meat in cooked organs, and therefore fat was included in the "edible portion" in Table 3. Cooked lamb edible portions ranged between $28.6 \mathrm{~g}$ (kidneys) and $713 \mathrm{~g}$ (stomachs). Similarly to the small mutton organs, edible portions of cooked lamb hearts, kidneys, spleens, lungs and tongues did not differ significantly in terms of weight in grams, ranging between $28.6 \mathrm{~g}$ (kidneys) and 259g (lungs). Furthermore there was a significant difference between the cooked edible portions of lamb livers (130g) and lamb intestines (896g). There was no significant difference between the cooked lamb livers and stomachs (714g) and also not between the intestines $(896 \mathrm{~g})$ and stomachs.

Yield factors presented in Table 2 for mutton organs ranged between 36.3\% (kidneys) and 76.3\% (livers). Yield factors for lamb organs in Table 2 ranged between 55.1\% (kidneys) and 83.8\% (livers). Although cooked lamb and mutton livers did not yield the largest edible portion in terms of weight they had the largest yield factor and thus had the lowest percentage cooking losses. This is consistent with what was found by the New Zealand study on the yield of cooked lamb organs (Purchas \& Wilkinson, 2013). Higher cooking losses were reported than observed in other South African studies reporting on yields and cooking losses of meat cuts.

\section{Proximate and mineral composition per $100 \mathrm{~g}$ raw and cooked lamb and mutton organ meats}

The results of the proximate analysis and the mineral content of raw mutton organs per 100g are presented in Table 3 and for raw lamb organs in Table 4. Significant differences were found between organs for all nutrients tested in both raw lamb and raw mutton 
organs. The results of the proximate analysis and the mineral content of cooked mutton organs are presented in Table 5 and for cooked lamb organs in Table 6.

Table 2: Cooking data and yield factors for mutton organ meats

\begin{tabular}{|c|c|c|c|c|c|c|}
\hline & \multicolumn{2}{|c|}{ Raw weight } & \multicolumn{2}{|c|}{ Cooked edible portion } & \multirow{2}{*}{$\begin{array}{l}\text { Yield } \\
\text { factor* }\end{array}$} & \multirow{2}{*}{$\begin{array}{c}\text { Cooking } \\
\text { loss }\end{array}$} \\
\hline \multicolumn{5}{|c|}{ Mutton } & & \\
\hline$n=3$ & g & $\pm s . d$ & g & $\pm s . d$ & $\%$ & $\%$ \\
\hline Intestines & $1837^{\mathrm{a}}$ & $( \pm 244)$ & $782^{b}$ & $( \pm 112)$ & 42.5 & 57.5 \\
\hline Lungs & $610^{b}$ & $( \pm 158)$ & $318^{\mathrm{cd}}$ & $( \pm 116)$ & 51.1 & 48.9 \\
\hline Hearts & $187^{b c}$ & $( \pm 7.44)$ & $132^{d}$ & $( \pm 16.6)$ & 70.4 & 29.6 \\
\hline Livers & $624^{b}$ & $( \pm 106)$ & $477^{b c}$ & $( \pm 100)$ & 76.3 & 23.7 \\
\hline Stomachs & $2189^{a}$ & $( \pm 223)$ & $1289^{b}$ & $( \pm 178)$ & 59.3 & 40.7 \\
\hline Kidneys & $80.0^{c}$ & $( \pm 4.87)$ & $29.1^{d}$ & $( \pm 2.50)$ & 36.3 & 63.7 \\
\hline Spleen & $109^{c}$ & $( \pm 8.21)$ & $65.0^{\mathrm{d}}$ & $( \pm 15.8)$ & 58.9 & 41.1 \\
\hline Tongue & $92.0^{c}$ & $( \pm 5.51)$ & $56.9^{d}$ & $( \pm 13.3)$ & 61.7 & 38.3 \\
\hline \multicolumn{7}{|c|}{ Note: Means with different superscripts in column differ significantly } \\
\hline \multicolumn{7}{|c|}{ Lamb } \\
\hline$n=3$ & g & $( \pm s . d)$ & g & $( \pm s . d)$ & $\%$ & $\%$ \\
\hline Intestines & $2009^{\mathrm{a}}$ & $( \pm 141)$ & $896^{\mathrm{a}}$ & $( \pm 107)$ & 44.5 & 55.5 \\
\hline Lungs & $459^{c}$ & $( \pm 9.9)$ & $259^{c}$ & $( \pm 23.8)$ & 56.4 & 43.6 \\
\hline Hearts & $192^{\mathrm{e}}$ & $( \pm 15.1)$ & $130^{c}$ & $( \pm 9.7)$ & 68.0 & 32.0 \\
\hline Livers & $696^{c}$ & $( \pm 97.2)$ & $583^{\mathrm{b}}$ & $( \pm 77.2)$ & 83.8 & 16.2 \\
\hline Stomachs & $1130^{b}$ & $( \pm 58.7)$ & $714^{\mathrm{ab}}$ & $( \pm 174)$ & 62.7 & 37.3 \\
\hline Kidneys & $52.1^{\mathrm{e}}$ & $( \pm 4.9)$ & $28.6^{\mathrm{c}}$ & $( \pm 8.1)$ & 55.1 & 44.9 \\
\hline Spleen & $72.0^{\mathrm{e}}$ & $( \pm 22.3)$ & $42.5^{c}$ & $( \pm 18.9)$ & 56.0 & 44.0 \\
\hline Tongue & $94.2^{\mathrm{e}}$ & $( \pm 13.7)$ & $70.0^{c}$ & $( \pm 6.8)$ & 75.0 & 25.0 \\
\hline \multicolumn{7}{|c|}{ Note: Means with different superscripts in column differ significantly } \\
\hline
\end{tabular}


Significant differences were found between raw mutton organs for moisture, ash, protein and fat values, as well as for all minerals tested (Table 3). Similarly for raw lamb, significant differences were found between organs for moisture, ash, protein and fat values, as well as for all minerals tested (Table 4).

Animal source foods contain good quality proteins in a bioavailable form. Referring to Table 5 the cooked mutton organs with the highest protein value per $100 \mathrm{~g}$ were kidneys $(32.7 \mathrm{~g} / 100 \mathrm{~g})$, with the other cooked mutton organs ranging between $15.3 \mathrm{~g} / 100 \mathrm{~g}$ (intestines) and $27.8 \mathrm{~g} / 100 \mathrm{~g}$ (spleen). Spleens were the cooked lamb organs with the highest amount of protein $(29.5 \mathrm{~g} / 100 \mathrm{~g})$, with the other organs ranging between $14.3 \mathrm{~g} / 100 \mathrm{~g}$ (intestines) and $24.8 \mathrm{~g} / 100 \mathrm{~g}$ (stomachs).

In terms of the mineral composition of cooked lamb and mutton organs, values differed significantly between all organs with the exception of magnesium in cooked lamb organs. No significant difference in magnesium content could be found between cooked organs (Table 6).

Values for iron and zinc in cooked lamb and mutton organ meats, which are nutrients of concern for the South African population (Shisana, et al., 2014), are presented in Table 5 and Table 6. The iron content of cooked mutton organs (Table 5) differed significantly between the different organs ranging from $1.69 \mathrm{mg} / 100 \mathrm{~g}$ (intestines) to $11.7 \mathrm{mg} / 100 \mathrm{~g}$ (spleens). The iron content of lamb organs (Table 6) differed to a lesser extent between the organs, ranging between $1.4 \mathrm{mg} / 100 \mathrm{~g}$ (intestines) and $22.8 \mathrm{mg}$ (spleen).

The phosphorus content of mutton organs ranged between $112 \mathrm{mg} / 100 \mathrm{~g}$ (intestines and stomachs) and $414 \mathrm{mg} / 100 \mathrm{~g}$ (spleens) and for lamb organs between $124 \mathrm{mg} / 100 \mathrm{~g}$ (intestines) and $423 \mathrm{mg} / 100 \mathrm{~g}$ (livers). Low levels of manganese, potassium, sodium and calcium were found in all lamb and mutton organs. 
Table 3: Proximate composition and mineral content of $100 \mathrm{~g}$ edible portion (without bone and cartilage) raw mutton organ meats

\begin{tabular}{|c|c|c|c|c|c|c|c|c|c|c|c|c|}
\hline & 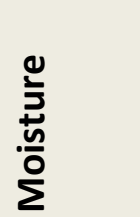 & $\frac{5}{4}$ & 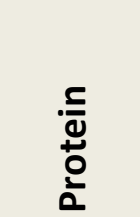 & 烍 & $\frac{\frac{E}{3}}{\frac{3}{d}}$ & $\begin{array}{l}\frac{n}{2} \\
\frac{0}{0} \\
\frac{c}{0} \\
\frac{0}{0} \\
\frac{c}{\alpha}\end{array}$ & 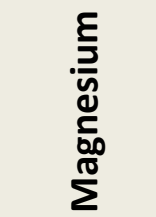 & 음 & 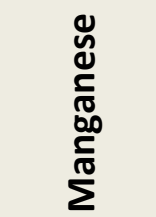 & $\stackrel{\text { Un }}{\stackrel{N}{N}}$ & 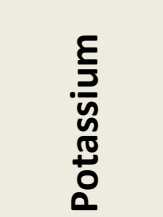 & 竞 \\
\hline & $\begin{array}{c}g / 100 g \\
( \pm s . d)\end{array}$ & $\begin{array}{c}g / 100 g \\
( \pm s . d)\end{array}$ & $\begin{array}{c}g / 100 g \\
( \pm s . d)\end{array}$ & $\begin{array}{c}g / 100 g \\
( \pm s . d)\end{array}$ & $\begin{array}{c}\mathrm{mg} / 100 \mathrm{~g} \\
( \pm \mathrm{s} . \mathrm{d})\end{array}$ & $\begin{array}{c}\mathrm{mg} / 100 \mathrm{~g} \\
( \pm s . d)\end{array}$ & $\begin{array}{c}\mathrm{mg} / 100 \mathrm{~g} \\
( \pm s . d)\end{array}$ & $\begin{array}{c}\mathrm{mg} / 100 \mathrm{~g} \\
( \pm s . d)\end{array}$ & $\begin{array}{c}\mathrm{mg} / 100 \mathrm{~g} \\
( \pm s . d)\end{array}$ & $\begin{array}{c}\mathrm{mg} / 100 \mathrm{~g} \\
( \pm s . d)\end{array}$ & $\begin{array}{c}\mathrm{mg} / 100 \mathrm{~g} \\
( \pm s . d)\end{array}$ & $\begin{array}{c}\mathrm{mg} / 100 \mathrm{~g} \\
( \pm s . d)\end{array}$ \\
\hline \multirow{2}{*}{ Intestines } & $64.5^{c d}$ & $0.28^{d}$ & $6.96^{\mathrm{e}}$ & $28.6^{a}$ & $11.0^{b}$ & $79.0^{d}$ & $14.67^{e}$ & $1.29^{b}$ & $0.04^{b}$ & $0.92^{d}$ & $88.0^{\mathrm{e}}$ & $39.6^{c}$ \\
\hline & $( \pm 6.12)$ & $( \pm 0.15)$ & $( \pm 1.29)$ & $( \pm 8.16)$ & $( \pm 3.50)$ & $( \pm 23.6)$ & $( \pm 4.00)$ & $( \pm 0.30)$ & $( \pm 0.03)$ & $( \pm 0.23)$ & $( \pm 28.1)$ & $( \pm 5.94)$ \\
\hline \multirow{2}{*}{ Lungs } & $79.3^{\mathrm{a}}$ & $1.08^{b}$ & $16.7^{b}$ & $2.41^{\mathrm{e}}$ & $8.00^{b}$ & $225^{a b c}$ & $17.0^{\mathrm{de}}$ & $7.76^{b}$ & $0.01^{b}$ & $1.70^{c}$ & $285^{b c}$ & $149^{\mathrm{a}}$ \\
\hline & $( \pm 0.60)$ & $( \pm 0.17)$ & $( \pm 0.61)$ & $( \pm 0.19)$ & $( \pm 1.30)$ & $( \pm 25.5)$ & $( \pm 3.00)$ & $( \pm 1.81)$ & $( \pm 0.01)$ & $( \pm 0.09)$ & $( \pm 20.2)$ & $( \pm 4.08)$ \\
\hline \multirow{2}{*}{ Hearts } & $70.3^{b}$ & $1.10^{b}$ & $16.3^{b}$ & $11.7^{\mathrm{cd}}$ & $7.60^{b}$ & $186^{b c d}$ & $23.1^{a b c}$ & $3.47^{b}$ & $0.03^{b}$ & $1.71^{c}$ & $256^{\mathrm{cd}}$ & $109^{b}$ \\
\hline & $( \pm 2.83)$ & $( \pm 0.16)$ & $( \pm 1.42)$ & $( \pm 2.65)$ & $( \pm 4.00)$ & $( \pm 16.9)$ & $( \pm 2.40)$ & $( \pm 0.28)$ & $( \pm 0.001)$ & $( \pm 0.17)$ & $( \pm 44.9)$ & $( \pm 28.4)$ \\
\hline \multirow{2}{*}{ Livers } & $69.9^{b c}$ & $1.87^{\mathrm{a}}$ & $19.9^{\mathrm{a}}$ & $4.33^{\mathrm{de}}$ & $6.33^{b}$ & $259^{\mathrm{ab}}$ & $26.8^{a}$ & $15.3^{b}$ & $0.28^{\mathrm{ab}}$ & $4.02^{a}$ & $334^{b}$ & $63.0^{c}$ \\
\hline & $( \pm 1.01)$ & $( \pm 0.60)$ & $( \pm 1.13)$ & $( \pm 0.72)$ & $( \pm 1.00)$ & $( \pm 183)$ & $( \pm 1.40)$ & $( \pm 2.93)$ & $( \pm 0.05)$ & $( \pm 0.39)$ & $( \pm 39.0)$ & $( \pm 23.6)$ \\
\hline \multirow{2}{*}{ Stomachs } & $70.9^{b}$ & $0.39^{d}$ & $10.3^{d}$ & $18.5^{b c}$ & $26.5^{a}$ & $88.2^{d}$ & $15.5^{\mathrm{de}}$ & $4.10^{b}$ & $0.78^{a}$ & $1.57^{c}$ & $134^{e}$ & $53.0^{c}$ \\
\hline & $( \pm 3.89)$ & $( \pm 0.08)$ & $( \pm 1.07)$ & $( \pm 3.97)$ & $( \pm 5.70)$ & $( \pm 14.6)$ & $( \pm 2.10)$ & $( \pm 3.67)$ & $( \pm 1.00)$ & $( \pm 0.28)$ & $( \pm 20.4)$ & $( \pm 7.62)$ \\
\hline \multirow{2}{*}{ Kidneys } & $80.5^{a}$ & $1.03^{b c}$ & $14.9^{b}$ & $3.03^{e}$ & $8.50^{b}$ & $224^{a b c}$ & $20.3^{b c d}$ & $2.97^{b}$ & $0.09^{b}$ & $1.87^{c}$ & $249^{c d}$ & $150^{\mathrm{a}}$ \\
\hline & $( \pm 1.07)$ & $( \pm 0.06)$ & $( \pm 0.80)$ & $( \pm 0.42)$ & $( \pm 0.80)$ & $( \pm 14.9)$ & $( \pm 1.20)$ & $( \pm 0.35)$ & $( \pm 0.01)$ & $( \pm 0.32)$ & $( \pm 30.0)$ & $( \pm 9.35)$ \\
\hline \multirow{2}{*}{ Spleen } & $77.6^{a}$ & $1.34^{\mathrm{b}}$ & $19.6^{\mathrm{a}}$ & $2.86^{\mathrm{e}}$ & $6.50^{b}$ & $317^{a}$ & $25.1^{\mathrm{ab}}$ & $97.4^{\mathrm{a}}$ & $0.02^{b}$ & $2.69^{b}$ & $464^{a}$ & $106^{b}$ \\
\hline & $( \pm 0.70)$ & $( \pm 0.08)$ & $( \pm 0.87)$ & $( \pm 0.26)$ & $( \pm 1.20)$ & $( \pm 45.8)$ & $( \pm 2.60)$ & $( \pm 43.6)$ & $( \pm 0.02)$ & $( \pm 0.13)$ & $( \pm 37.9)$ & $( \pm 4.79)$ \\
\hline \multirow{2}{*}{ Tongues } & $64.1^{d}$ & $0.61^{c d}$ & $12.9^{c}$ & $21.7^{\mathrm{ab}}$ & $6.83^{d}$ & $129^{\mathrm{cd}}$ & $20.2^{c d}$ & $1.52^{b}$ & $0.01^{b}$ & $1.65^{a}$ & $218^{a}$ & $102^{b}$ \\
\hline & $( \pm 3.30)$ & $( \pm 0.09)$ & $( \pm 0.74)$ & $( \pm 3.57)$ & $( \pm 0.98)$ & $( \pm 8.6)$ & $( \pm 3.70)$ & $( \pm 0.17)$ & $( \pm 0.02)$ & $( \pm 0.16)$ & $( \pm 20.8)$ & $( \pm 10.8)$ \\
\hline P-value & $<0.001$ & $<0.001$ & $<0.001$ & $<0.001$ & $<0.001$ & $<0.001$ & $<0.001$ & $<0.001$ & 0.007 & $<0.001$ & $<0.001$ & $<0.001$ \\
\hline
\end{tabular}

Note: Means with different superscripts in a column differ significantly 
Table 4: Proximate composition and mineral content of $100 \mathrm{~g}$ edible portion (without bone and cartilage) raw lamb organ meats

\begin{tabular}{|c|c|c|c|c|c|c|c|c|c|c|c|c|}
\hline & 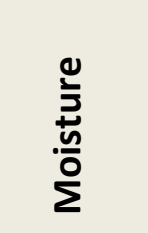 & $\frac{5}{\frac{1}{\alpha}}$ & 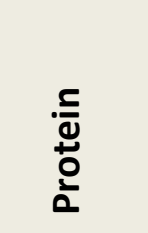 & 蒈 & $\frac{\frac{E}{J}}{\frac{D}{\sqrt{U}}}$ & $\begin{array}{l}\text { ำ } \\
\frac{0}{0} \\
\frac{c}{0} \\
\frac{0}{0} \\
\frac{c}{a}\end{array}$ & 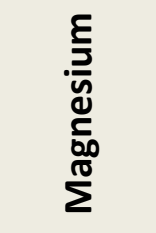 & 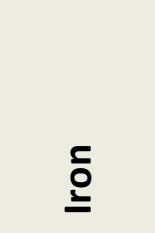 & 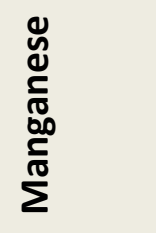 & 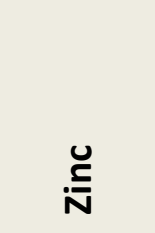 & 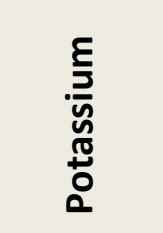 & $\begin{array}{l}\frac{\varepsilon}{\partial} \\
\frac{7}{\overline{0}} \\
\text { ம }\end{array}$ \\
\hline & $\begin{array}{c}g / 100 g \\
( \pm s . d)\end{array}$ & $\begin{array}{l}g / 100 \mathrm{~g} \\
( \pm s . d)\end{array}$ & $\begin{array}{l}g / 100 \mathrm{~g} \\
( \pm s . d)\end{array}$ & $\begin{array}{l}g / 100 \mathrm{~g} \\
( \pm s . d)\end{array}$ & $\begin{array}{c}\mathrm{mg} / 100 \mathrm{~g} \\
\text { (士s.d) }\end{array}$ & $\begin{array}{c}\mathrm{mg} / 100 \mathrm{~g} \\
\text { (士s.d) }\end{array}$ & $\begin{array}{c}\mathrm{mg} / 100 \mathrm{~g} \\
\text { ( } \pm \text { s.d) }\end{array}$ & $\begin{array}{c}\mathrm{mg} / 100 \mathrm{~g} \\
\text { ( } \pm \mathrm{s} . \mathrm{d})\end{array}$ & $\begin{array}{c}\mathrm{mg} / 100 \mathrm{~g} \\
\text { ( } \pm \mathrm{s.d})\end{array}$ & $\begin{array}{c}\mathrm{mg} / 100 \mathrm{~g} \\
\text { ( } \pm \mathrm{s} . \mathrm{d})\end{array}$ & $\begin{array}{c}\mathrm{mg} / 100 \mathrm{~g} \\
\text { (士s.d) }\end{array}$ & $\begin{array}{c}\mathrm{mg} / 100 \mathrm{~g} \\
\text { ( } \pm \mathrm{s} . \mathrm{d})\end{array}$ \\
\hline \multirow{2}{*}{ Intestines } & $55.2^{\text {cd }}$ & $0.46^{\mathrm{e}}$ & $7.01^{\mathrm{e}}$ & $26.5^{\mathrm{a}}$ & $9.33^{\mathrm{ab}}$ & $95.0^{\mathrm{e}}$ & $11.3^{\mathrm{e}}$ & $1.37^{c}$ & $0.08^{b}$ & $1.00^{\mathrm{e}}$ & $94.3^{d}$ & $43.0^{\mathrm{e}}$ \\
\hline & $( \pm 7.16)$ & $( \pm 0.19)$ & $( \pm 1.41)$ & $( \pm 7.41)$ & $( \pm 3.14)$ & $( \pm 16.2)$ & $( \pm 1.75)$ & $( \pm 0.18)$ & $( \pm 0.04)$ & $( \pm 0.11)$ & $( \pm 13.0)$ & $( \pm 1.79)$ \\
\hline \multirow{2}{*}{ Lungs } & $74.1^{a}$ & $1.05^{b c}$ & $17.3^{\mathrm{ab}}$ & $2.50^{d}$ & $7.00^{b c}$ & $164^{d}$ & $15.7^{d}$ & $15.8^{\mathrm{ab}}$ & $0.02^{c d}$ & $1.77^{\mathrm{cd}}$ & $252^{c}$ & $166^{\mathrm{a}}$ \\
\hline & $( \pm 0.69)$ & $( \pm 0.35)$ & $( \pm 0.72)$ & $( \pm 0.84)$ & $( \pm 0.00)$ & $( \pm 44.7)$ & $( \pm 1.21)$ & $( \pm 5.44)$ & $( \pm 0.01)$ & $( \pm 0.24)$ & $( \pm 20.9)$ & $( \pm 14.2)$ \\
\hline \multirow{2}{*}{ Hearts } & $65.1^{b}$ & $0.87^{\text {cd }}$ & $17.2^{\mathrm{ab}}$ & $11.8^{\mathrm{bc}}$ & $4.83^{c}$ & $199^{\text {cd }}$ & $23.2^{a b}$ & $3.93^{c}$ & $0.00^{d}$ & $1.83^{C}$ & $280^{b c}$ & $118^{b c}$ \\
\hline & $( \pm 0.32)$ & $( \pm 0.52)$ & $( \pm 1.26)$ & $( \pm 0.62)$ & $( \pm 1.17)$ & $( \pm 16.2)$ & $( \pm 2.40)$ & $( \pm 0.76)$ & $( \pm 0.00)$ & $( \pm 0.06)$ & $( \pm 30.0)$ & $( \pm 13.8)$ \\
\hline \multirow{2}{*}{ Livers } & $61.2^{\mathrm{bc}}$ & $1.40^{\mathrm{a}}$ & $18.5^{\mathrm{a}}$ & $8.90^{c d}$ & $5.50^{c}$ & $349^{a}$ & $21.2^{\mathrm{abc}}$ & $5.11^{b c}$ & $0.15^{\mathrm{a}}$ & $3.02^{a}$ & $310^{b}$ & $66.8^{\text {de }}$ \\
\hline & $( \pm 3.42)$ & $( \pm 0.12)$ & $( \pm 0.50)$ & $( \pm 5.28)$ & $( \pm 0.55)$ & $( \pm 24.0)$ & $( \pm 0.75)$ & $( \pm 0.51)$ & $( \pm 0.02)$ & $( \pm 0.19)$ & $( \pm 9.14)$ & $( \pm 5.78)$ \\
\hline \multirow{2}{*}{ Stomachs } & $49.6^{d}$ & $0.43^{\mathrm{e}}$ & $10.0^{d}$ & $15.7^{\mathrm{bc}}$ & $11.5^{\mathrm{a}}$ & $92.0^{\mathrm{e}}$ & $11.7^{\mathrm{e}}$ & $2.27^{c}$ & $0.05^{b c}$ & $1.48^{\mathrm{d}}$ & $129^{d}$ & $57.7^{\mathrm{de}}$ \\
\hline & $( \pm 2.15)$ & $( \pm 0.02)$ & $( \pm 0.91)$ & $( \pm 1.78)$ & $( \pm 2.26)$ & $( \pm 5.56)$ & $( \pm 0.52)$ & $( \pm 0.83)$ & $( \pm 0.03)$ & $( \pm 0.07)$ & $( \pm 7.10)$ & $( \pm 7.79)$ \\
\hline \multirow{2}{*}{ Kidneys } & $65.8^{\mathrm{b}}$ & $1.10^{\mathrm{b}}$ & $15.2^{c}$ & $3.20^{d}$ & $6.33^{b c}$ & $227^{b c}$ & $18.0^{c}$ & $3.42^{c}$ & $0.09^{b}$ & $2.06^{\mathrm{C}}$ & $269^{b c}$ & $155^{a}$ \\
\hline & $( \pm 0.21)$ & $( \pm 0.08)$ & $( \pm 0.34)$ & $( \pm 0.25)$ & $( \pm 0.52)$ & $( \pm 10.7)$ & $( \pm 0.98)$ & $( \pm 0.20)$ & $( \pm 0.01)$ & $( \pm 0.04)$ & $( \pm 6.90)$ & $( \pm 4.51)$ \\
\hline \multirow{2}{*}{ Spleen } & $67.1^{\mathrm{ab}}$ & $1.18^{\mathrm{b}}$ & $17.9^{\mathrm{ab}}$ & $2.00^{d}$ & $4.33^{c}$ & $258^{b}$ & $20.0^{b c}$ & $19.7^{\mathrm{a}}$ & $0.02^{\text {cd }}$ & $2.41^{\mathrm{b}}$ & $403^{a}$ & $95.0^{c}$ \\
\hline & $( \pm 0.10)$ & $( \pm 0.08)$ & $( \pm 0.36)$ & $( \pm 0.12)$ & $( \pm 0.82)$ & $( \pm 32.2)$ & $( \pm 0.89)$ & $( \pm 13.9)$ & $( \pm .0 .001)$ & $( \pm 0.25)$ & $( \pm 18.4)$ & $( \pm 4.34)$ \\
\hline \multirow{2}{*}{ Tongues } & $63.7^{b}$ & $0.83^{d}$ & $16.0^{b c}$ & $17.7^{b}$ & $7.33^{b c}$ & $171^{d}$ & $24.2^{\mathrm{a}}$ & $1.77^{c}$ & $0.04^{\mathrm{cd}}$ & $1.99^{\mathrm{C}}$ & $298^{b}$ & $112^{\mathrm{bc}}$ \\
\hline & $( \pm 2.82)$ & $( \pm 0.07)$ & $( \pm 1.34)$ & $( \pm 16.75)$ & $( \pm 7.0)$ & $( \pm 12.6)$ & $( \pm 2.64)$ & $( \pm 0.16)$ & $( \pm 0.003)$ & $( \pm 0.19)$ & $( \pm 36.5)$ & $( \pm 15.4)$ \\
\hline P-value & $<0.001$ & $<0.001$ & $<0.001$ & $<0.001$ & $<0.001$ & $<0.001$ & $<0.001$ & $<0.001$ & $<0.001$ & $<0.001$ & $<0.001$ & $<0.001$ \\
\hline
\end{tabular}


Table 5: Proximate composition and mineral content of $100 \mathrm{~g}$ edible portion (without bone and cartilage) cooked mutton organ meats

\begin{tabular}{|c|c|c|c|c|c|c|c|c|c|c|c|c|}
\hline & 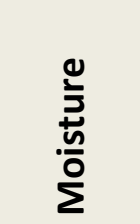 & $\frac{5}{\frac{1}{4}}$ & 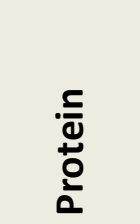 & 毞 & $\frac{\xi}{\frac{3}{U}}$ & $\begin{array}{l}\text { ă } \\
\frac{0}{0} \\
\frac{0}{0} \\
\frac{0}{0} \\
\frac{c}{0}\end{array}$ & 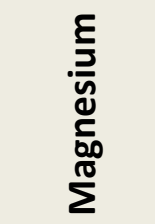 & 음 & 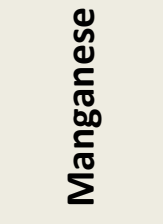 & $\underset{\mathbf{N}}{\stackrel{u}{N}}$ & 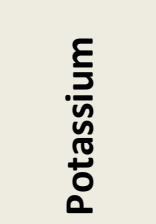 & $\begin{array}{l}\frac{\xi}{3} \\
\frac{3}{8} \\
\stackrel{\circ}{\circ}\end{array}$ \\
\hline & $\begin{array}{c}g / 100 g \\
( \pm s . d)\end{array}$ & $\begin{array}{c}g / 100 g \\
( \pm s . d)\end{array}$ & $\begin{array}{c}g / 100 g \\
( \pm s . d)\end{array}$ & $\begin{array}{c}g / 100 \mathrm{~g} \\
( \pm s . d)\end{array}$ & $\begin{array}{c}\mathrm{mg} / 100 \mathrm{~g} \\
( \pm \mathrm{s} . \mathrm{d})\end{array}$ & $\begin{array}{c}\mathrm{mg} / 100 \mathrm{~g} \\
( \pm \mathrm{s} . \mathrm{d})\end{array}$ & $\begin{array}{c}\mathrm{mg} / 100 \mathrm{~g} \\
( \pm s . d)\end{array}$ & $\begin{array}{c}\mathrm{mg} / 100 \mathrm{~g} \\
( \pm \mathrm{s} . \mathrm{d})\end{array}$ & $\begin{array}{c}\mathrm{mg} / 100 \mathrm{~g} \\
( \pm s . d)\end{array}$ & $\begin{array}{c}\mathrm{mg} / 100 \mathrm{~g} \\
( \pm \mathrm{s} . \mathrm{d})\end{array}$ & $\begin{array}{c}\mathrm{mg} / 100 \mathrm{~g} \\
( \pm \mathrm{s} . \mathrm{d})\end{array}$ & $\begin{array}{c}\mathrm{mg} / 100 \mathrm{~g} \\
( \pm \mathrm{s} . \mathrm{d})\end{array}$ \\
\hline \multirow[t]{2}{*}{ Intestines } & $48.2^{d}$ & $0.74^{d}$ & $15.3^{\mathrm{d}}$ & $37.9^{\mathrm{a}}$ & $16.6^{b}$ & $112^{\mathrm{C}}$ & $16.9^{\mathrm{cd}}$ & $1.69^{\mathrm{e}}$ & $0.0002^{\mathrm{e}}$ & $2.55^{b}$ & $50.2^{d}$ & $29.5^{e}$ \\
\hline & $( \pm 6.68)$ & $( \pm 0.23)$ & $( \pm 2.40)$ & $( \pm 7.71)$ & $( \pm 0.80)$ & $( \pm 40.6)$ & $( \pm 2.90)$ & $( \pm 0.12)$ & $( \pm 0.00)$ & $( \pm 0.22)$ & $( \pm 16.8)$ & $( \pm 7.68)$ \\
\hline \multirow[t]{2}{*}{ Lungs } & $71.1^{\mathrm{a}}$ & $1.21^{\mathrm{c}}$ & $23.2^{\mathrm{bc}}$ & $3.97^{d}$ & $10.99^{b c}$ & $250^{b}$ & $19.4^{\mathrm{bcd}}$ & $10.7^{\mathrm{a}}$ & $0.0010^{a}$ & $2.62^{b}$ & $285^{b c}$ & $190^{b}$ \\
\hline & $( \pm 0.94)$ & $( \pm 0.11)$ & $( \pm 1.11)$ & $( \pm 0.87)$ & $( \pm 1.60)$ & $( \pm 18.6)$ & $( \pm 1.70)$ & $( \pm 0.26)$ & $( \pm 0.00)$ & $( \pm 0.18)$ & $( \pm 31.3)$ & $( \pm 37.6)$ \\
\hline \multirow[t]{2}{*}{ Hearts } & $57.6^{\mathrm{bc}}$ & $1.34^{\mathrm{bc}}$ & $20.4^{\mathrm{cd}}$ & $20.2^{c}$ & $6.00^{c}$ & $223^{b}$ & $24.8^{\mathrm{ab}}$ & $4.54^{c}$ & $0.0005^{c}$ & $2.74^{b}$ & $275^{b c}$ & $97.5^{\mathrm{cd}}$ \\
\hline & $( \pm 4.98)$ & $( \pm 0.28)$ & $( \pm 3.57)$ & $( \pm 1.89)$ & $( \pm 2.30)$ & $( \pm 38.0)$ & $( \pm 4.40)$ & $( \pm 1.13)$ & $( \pm 0.0001)$ & $( \pm 0.42)$ & $( \pm 62.0)$ & $( \pm 18.9)$ \\
\hline \multirow[t]{2}{*}{ Livers } & $64.5^{\mathrm{ab}}$ & $1.59^{b c}$ & $23.1^{b c}$ & $6.27^{d}$ & $5.60^{c}$ & $399^{a}$ & $26.2^{\mathrm{ab}}$ & $7.96^{b}$ & $0.0008^{d}$ & $4.38^{a}$ & $326^{b c}$ & $78.7^{\text {cde }}$ \\
\hline & $( \pm 2.00)$ & $( \pm 0.12)$ & $( \pm 0.20)$ & $( \pm 0.93)$ & $( \pm 1.50)$ & $( \pm 7.00)$ & $( \pm 1.60)$ & $( \pm 1.11)$ & $( \pm 0.0001)$ & $( \pm 0.54)$ & $( \pm 19.2)$ & $( \pm 8.45)$ \\
\hline \multirow[t]{2}{*}{ Stomachs } & $53.1^{\mathrm{cd}}$ & $0.61^{d}$ & $17.8^{d}$ & $27.3^{b c}$ & $24.6^{a}$ & $112^{\mathrm{C}}$ & $15.9^{d}$ & $2.70^{\mathrm{de}}$ & $0.0002^{\mathrm{de}}$ & $3.37^{\mathrm{ab}}$ & $104^{d}$ & $58.7^{\text {de }}$ \\
\hline & $( \pm 6.58)$ & $( \pm 0.12)$ & $( \pm 2.79)$ & $( \pm 3.25)$ & $( \pm 7.90)$ & $( \pm 33.6)$ & $( \pm 4.60)$ & $( \pm 1.00)$ & $( \pm 0.0001)$ & $( \pm 1.35)$ & $( \pm 32.7)$ & $( \pm 17.7)$ \\
\hline \multirow[t]{2}{*}{ Kidneys } & $57.2^{\mathrm{bcd}}$ & $2.36^{\mathrm{a}}$ & $32.7^{a}$ & $7.77^{e}$ & $15.6^{b}$ & $400^{a}$ & $30.7^{a}$ & $4.34^{\mathrm{cd}}$ & $0.0004^{\mathrm{cd}}$ & $4.49^{a}$ & $279^{b c}$ & $270^{a}$ \\
\hline & $( \pm 1.49)$ & $( \pm 0.18)$ & $( \pm 3.06)$ & $( \pm 1.15)$ & $( \pm 0.75)$ & $( \pm 36.3)$ & $( \pm 3.40)$ & $( \pm 0.67)$ & $( \pm 0.0006)$ & $( \pm 0.17)$ & $( \pm 57.7)$ & $( \pm 57.5)$ \\
\hline \multirow[t]{2}{*}{ Spleen } & $66.2^{\mathrm{ab}}$ & $1.69^{b c}$ & $27.8^{\mathrm{ab}}$ & $5.23^{\mathrm{e}}$ & $6.00^{c}$ & $414^{a}$ & $31.4^{\mathrm{a}}$ & $11.7^{\mathrm{a}}$ & $0.0011^{a}$ & $3.61^{a b}$ & $472^{a}$ & $112^{\mathrm{cd}}$ \\
\hline & $( \pm 3.10)$ & $( \pm 0.21)$ & $( \pm 2.83)$ & $( \pm 0.68)$ & $( \pm 0.60)$ & $( \pm 41.8)$ & $( \pm 2.70)$ & $( \pm 0.96)$ & $( \pm 0.0009)$ & $( \pm 0.38)$ & $( \pm 51.1)$ & $( \pm 10.2)$ \\
\hline \multirow[t]{2}{*}{ Tongues } & $52.6^{\mathrm{cd}}$ & $0.71^{d}$ & $15.8^{d}$ & $33.2^{\mathrm{ab}}$ & $8.70^{c}$ & $142^{c}$ & $23.3^{b c}$ & $1.81^{\mathrm{e}}$ & $0.0002^{\mathrm{e}}$ & $2.91^{b}$ & $235^{c}$ & $122^{c}$ \\
\hline & $( \pm 8.72)$ & $( \pm 0.18)$ & $( \pm 1.51)$ & $( \pm 7.47)$ & $( \pm 0.60)$ & $( \pm 15.4)$ & $( \pm 2.61)$ & $( \pm 0.34)$ & $( \pm 0.00003)$ & $( \pm 0.36)$ & $( \pm 22.7)$ & $( \pm 13.0)$ \\
\hline P-value & $<0.001$ & $<0.001$ & $<0.001$ & $<0.001$ & $<0.001$ & $<0.001$ & $<0.001$ & $<0.001$ & $<0.001$ & $<0.001$ & $<0.001$ & $<0.001$ \\
\hline
\end{tabular}


Table 6: Proximate composition and mineral content of $100 \mathrm{~g}$ edible portion (without bone and cartilage) cooked lamb organ meats

\begin{tabular}{|c|c|c|c|c|c|c|c|c|c|c|c|c|}
\hline & 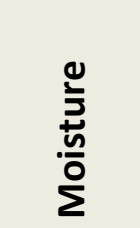 & $\frac{5}{4}$ & $\begin{array}{l}\frac{5}{\mathbb{N}} \\
\text { ơ } \\
\frac{0}{2}\end{array}$ & 華 & $\frac{\frac{\varepsilon}{3}}{\frac{\bar{J}}{\sqrt{0}}}$ & 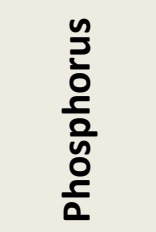 & 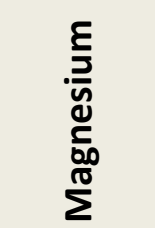 & $\underline{\underline{0}}$ & 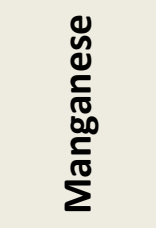 & $\stackrel{\text { In }}{N}$ & 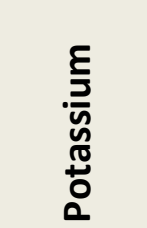 & $\begin{array}{l}\xi \\
\frac{\xi}{\bar{z}} \\
\dot{0}\end{array}$ \\
\hline & $\begin{array}{c}g / 100 \mathrm{~g} \\
( \pm s . d)\end{array}$ & $\begin{array}{c}g / 100 g \\
( \pm s . d)\end{array}$ & $\begin{array}{c}g / 100 \mathrm{~g} \\
( \pm s . d)\end{array}$ & $\begin{array}{c}g / 100 g \\
( \pm s . d)\end{array}$ & $\begin{array}{c}\mathrm{mg} / 100 \mathrm{~g} \\
( \pm s . d)\end{array}$ & $\begin{array}{c}\mathrm{mg} / 100 \mathrm{~g} \\
( \pm s . d)\end{array}$ & $\begin{array}{c}\mathrm{mg} / 100 \mathrm{~g} \\
( \pm \mathrm{s} . \mathrm{d})\end{array}$ & $\begin{array}{c}\mathrm{mg} / 100 \mathrm{~g} \\
( \pm \mathrm{s} . \mathrm{d})\end{array}$ & $\begin{array}{c}\mathrm{mg} / 100 \mathrm{~g} \\
( \pm \mathrm{s} . \mathrm{d})\end{array}$ & $\begin{array}{c}\mathrm{mg} / 100 \mathrm{~g} \\
( \pm \mathrm{s} . \mathrm{d})\end{array}$ & $\begin{array}{c}\mathrm{mg} / 100 \mathrm{~g} \\
( \pm \mathrm{s} . \mathrm{d})\end{array}$ & $\begin{array}{c}\mathrm{mg} / 100 \mathrm{~g} \\
( \pm s . d)\end{array}$ \\
\hline \multirow[t]{2}{*}{ Intestines } & $55.2^{\text {cd }}$ & $0.67^{c}$ & $14.3^{d}$ & $31.2^{\mathrm{a}}$ & $18.6^{\mathrm{b}}$ & $124^{\mathrm{e}}$ & $21.9^{a}$ & $1.40^{c}$ & $0.01^{c}$ & $2.60^{c}$ & $75.1^{d}$ & $38.4^{f}$ \\
\hline & $( \pm 1.81)$ & $( \pm 0.37)$ & $( \pm 2.22)$ & $( \pm 4.06)$ & $( \pm 8.79)$ & $( \pm 16.6)$ & $( \pm 8.80)$ & $( \pm 0.28)$ & $( \pm 0.01)$ & $( \pm 0.33)$ & $( \pm 30.0)$ & $( \pm 13.2)$ \\
\hline \multirow[t]{2}{*}{ Lungs } & $74.1^{\mathrm{a}}$ & $1.46^{\mathrm{ab}}$ & $21.1^{b c}$ & $6.53^{b}$ & $8.90^{b}$ & $271^{c}$ & $22.2^{\mathrm{a}}$ & $8.37^{b}$ & $0.00^{c}$ & $2.59^{c}$ & $298^{b}$ & $160^{b}$ \\
\hline & $( \pm 0.45)$ & $( \pm 0.19)$ & $( \pm 0.67)$ & $( \pm 5.51)$ & $( \pm 0.78)$ & $( \pm 18.1)$ & $( \pm 3.30)$ & $( \pm 0.41)$ & $( \pm 000)$ & $( \pm 0.19)$ & $( \pm 13.2)$ & $( \pm 24.5)$ \\
\hline \multirow[t]{2}{*}{ Hearts } & $65.1^{b}$ & $1.46^{\mathrm{ab}}$ & $19.3^{\mathrm{cd}}$ & $13.5^{b}$ & $5.12^{b}$ & $195^{d}$ & $29.0^{a}$ & $3.84^{b c}$ & $0.044^{c}$ & $2.49^{c}$ & $261^{b}$ & $101^{c d}$ \\
\hline & $( \pm 3.29)$ & $( \pm 0.54)$ & $( \pm 1.81)$ & $( \pm 4.59)$ & $( \pm 0.28)$ & $( \pm 18.5)$ & $( \pm 9.20)$ & $( \pm 0.19)$ & $( \pm 0.14)$ & $( \pm 0.15)$ & $( \pm 13.6)$ & $( \pm 5.91)$ \\
\hline \multirow[t]{2}{*}{ Livers } & $61.2^{\mathrm{bc}}$ & $1.78^{\mathrm{a}}$ & $23.6^{b c}$ & $8.39^{b}$ & $5.03^{b}$ & $423^{a}$ & $28.3^{a}$ & $6.07^{b c}$ & $0.27^{a}$ & $4.17^{a}$ & $315^{b}$ & $70.8^{e}$ \\
\hline & $( \pm 3.97)$ & $( \pm 0.18)$ & $( \pm 0.39)$ & $( \pm 4.49)$ & $( \pm 0.26)$ & $( \pm 18.8)$ & $( \pm 10.2)$ & $( \pm 0.82)$ & $( \pm 0.03)$ & $( \pm 0.11)$ & $( \pm 26.2)$ & $( \pm 6.78)$ \\
\hline \multirow[t]{2}{*}{ Stomachs } & $49.6^{d}$ & $0.87^{b c}$ & $24.8^{\mathrm{ab}}$ & $29.9^{a}$ & $52.7^{\mathrm{a}}$ & $170^{\mathrm{de}}$ & $25.3^{\mathrm{a}}$ & $4.85^{b c}$ & $0.19^{b}$ & $3.90^{\mathrm{a}}$ & $155^{c}$ & $79.5^{\mathrm{de}}$ \\
\hline & $( \pm 9.00)$ & $( \pm 0.20)$ & $( \pm 5.75)$ & $( \pm 6.50)$ & $( \pm 17.4)$ & $( \pm 49.0)$ & $( \pm 8.30)$ & $( \pm 0.99)$ & $( \pm 0.07)$ & $( \pm 0.81)$ & $( \pm 38.1)$ & $( \pm 20.2)$ \\
\hline \multirow[t]{2}{*}{ Kidneys } & $65.8^{\mathrm{b}}$ & $1.45^{\mathrm{a}}$ & $24.4^{\mathrm{abc}}$ & $12.1^{b}$ & $9.38^{b}$ & $330^{b}$ & $30.6^{a}$ & $4.44^{b c}$ & $0.05^{c}$ & $3.67^{a}$ & $310^{b}$ & $234^{a}$ \\
\hline & $( \pm 3.92)$ & $( \pm 0.15)$ & $( \pm 0.66)$ & $( \pm 0.84)$ & $( \pm 1.63)$ & $( \pm 5.80)$ & $( \pm 3.61)$ & $( \pm 0.83)$ & $( \pm 0.03)$ & $( \pm 0.35)$ & $( \pm 20.0)$ & $( \pm 13.9)$ \\
\hline \multirow[t]{2}{*}{ Spleen } & $67.1^{\mathrm{ab}}$ & $2.02^{\mathrm{a}}$ & $29.5^{a}$ & $6.62^{b}$ & $7.57^{b}$ & $406^{a}$ & $30.8^{a}$ & $22.8^{a}$ & $0.00^{c}$ & $3.60^{\mathrm{ab}}$ & $409^{a}$ & $112^{c}$ \\
\hline & $( \pm 1.45)$ & $( \pm 0.27)$ & $( \pm 1.68)$ & $( \pm 0.80)$ & $( \pm 1.36)$ & $( \pm 31.2)$ & $( \pm 2.70)$ & $( \pm 8.98)$ & $( \pm 0.00)$ & $( \pm 0.25)$ & $( \pm 45.4)$ & $( \pm 6.22)$ \\
\hline \multirow[t]{2}{*}{ Tongues } & $63.7^{b}$ & $0.78^{c}$ & $19.2^{\text {cd }}$ & $16.8^{b}$ & $17.7^{b}$ & $184^{d}$ & $24.0^{a}$ & $1.50^{\mathrm{bc}}$ & $0.00^{c}$ & $2.83^{\mathrm{ab}}$ & $276^{\mathrm{b}}$ & $102^{\mathrm{cd}}$ \\
\hline & $( \pm 4.76)$ & $( \pm 0.08)$ & $( \pm 1.49)$ & $( \pm 4.04)$ & $( \pm 10.8)$ & $( \pm 29.4)$ & $( \pm 1.50)$ & $( \pm 0.17)$ & $( \pm 0.00)$ & $( \pm 0.94)$ & $( \pm 31.4)$ & $( \pm 8.49)$ \\
\hline P-value & $<0.001$ & $<0.001$ & $<0.001$ & $<0.001$ & $<0.001$ & $<0.001$ & 0.132 & $<0.001$ & $<0.001$ & $<0.001$ & $<0.001$ & $<0.001$ \\
\hline
\end{tabular}


Potential nutritional contribution of cooked lamb and mutton organs per recommended serving

Nutrient reference values (NRVs) as per R429 amendment of the Foodstuffs, Cosmetics and Disinfectants Act (54/1972) (DOH, 2014), are presented in Table 7 together with the percentage contribution of a portion of each lamb and mutton organ to the NRV of each of the nutrients analysed as well as the nutritional content claims that can be made in accordance with the Foodstuffs, Cosmetics and Disinfectants Act (54/1972) (DOH, 2014). The recommended portion size for meat according to the South African Food-Based Dietary Guidelines is 90g edible portion (Schönfeldt, Pretorius, \& Hall, 2013).

NRVs, as presented in Table 7, are calculated for individuals from 37 months of age and older (DOH, 2014). By comparing the nutrient data found in this study, with the recommended NRVs, the' potential nutritional contribution per $90 \mathrm{~g}$ serving of these organs can be determined. For example $90 \mathrm{~g}$ of mutton kidneys, containing $29.4 \mathrm{~g}$ of crude protein may contribute up to $52 \%$ of an adult's daily protein allowance of $56 \mathrm{~g}$. According to the Foodstuffs, Cosmetics and Disinfectants Act (54/1972) a food product must contain between $15 \%$ and $30 \%$ of a given nutrient in order to be able to make the claim that a serving of that food product is "a source of" that given nutrient (DOH, 2014). Furthermore a food product must contain between $30 \%$ and $60 \%$ of a nutrient per serving to qualify as being "high in" that specific nutrient and more than $60 \%$ per serving for it to be "very high" or an "excellent source" of a certain nutrient (DOH, 2014). Based on these claim guidelines together with the values presented in Table 7, all lamb and mutton organ meats can make a significant nutritional contribution (whether it is a "source of" at least one nutrient or an "excellent source" of another). The different claims that can be made regarding lamb and mutton organ meats are presented in Table 7. 


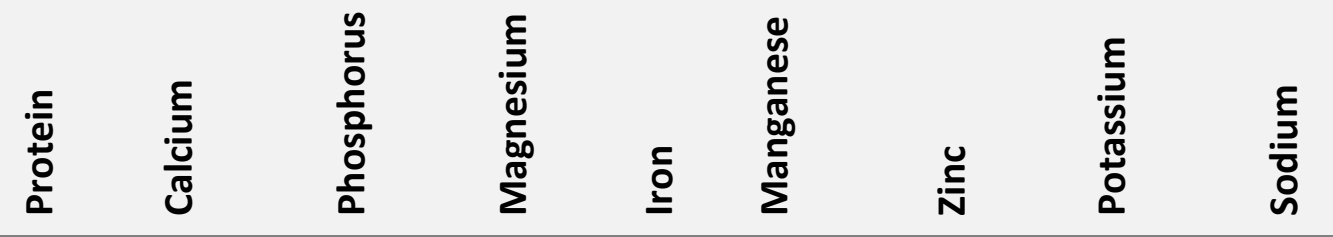

\begin{tabular}{|c|c|c|c|c|c|c|c|c|c|}
\hline $\mathbf{N R V}^{\prime}$ & $56 g$ & $1300 \mathrm{mg}$ & $1250 \mathrm{mg}$ & $365 \mathrm{mg}$ & $13 \mathrm{mg}$ & $2.3 \mathrm{mg}$ & $10 \mathrm{mg}$ & $4700 \mathrm{mg}$ & $2000 \mathrm{mg}$ \\
\hline Mutton & \multicolumn{9}{|c|}{$\%$ of NRV per $90 \mathrm{~g}$ serving ${ }^{\text {IIII }}$} \\
\hline Intestines & $25^{\mathrm{IV}}$ & 11 & 8 & 0 & 12 & 0 & $23^{\mathrm{IV}}$ & 1 & 1 \\
\hline Lungs & $37^{v}$ & 7 & $18^{\mathrm{IV}}$ & 0 & $74^{\mathrm{vl}}$ & 0 & $24^{\mathrm{IV}}$ & 5 & 9 \\
\hline Hearts & $33^{v}$ & 0 & $16^{\mathrm{IV}}$ & 0 & $31^{v}$ & 0 & $25^{\mathrm{IV}}$ & 5 & 4 \\
\hline Livers & $37^{v}$ & 0 & $29^{\mathrm{IV}}$ & 0 & $55^{\mathrm{v}}$ & 0 & $39^{v}$ & 6 & 4 \\
\hline Stomachs & $29^{\mathrm{IV}}$ & 2 & 8 & 0 & $19^{\mathrm{IV}}$ & 0 & $30^{v}$ & 2 & 3 \\
\hline Kidneys & $52^{v}$ & 1 & $29^{\mathrm{IV}}$ & 0 & $30^{v}$ & 0 & $40^{v}$ & 5 & 12 \\
\hline Spleen & $45^{v}$ & 0 & $30^{v}$ & 0 & $81^{\mathrm{vl}}$ & 0 & $32^{v}$ & 9 & 5 \\
\hline Tongues & $25^{\mathrm{IV}}$ & 1 & 10 & 0 & 13 & 0 & $26^{\mathrm{IV}}$ & 4 & 5 \\
\hline \multicolumn{4}{|l|}{ Lamb } & \multicolumn{6}{|c|}{$\%$ of NRV per $90 \mathrm{~g}$ serving ${ }^{\text {III }}$} \\
\hline Intestines & $23^{\mathrm{IV}}$ & 1 & 9 & 0 & 10 & 0 & $23^{\mathrm{IV}}$ & 1 & 2 \\
\hline Lungs & $34^{v}$ & 1 & $19^{\mathrm{IV}}$ & 0 & $58^{v}$ & 0 & $23^{\mathrm{IV}}$ & 6 & 7 \\
\hline Hearts & $31^{v}$ & 0 & 14 & 0 & $27^{\mathrm{IV}}$ & 2 & $22^{\mathrm{IV}}$ & 5 & 5 \\
\hline Livers & $38^{v}$ & 0 & $30^{v}$ & 0 & $42^{v}$ & 10 & $38^{v}$ & 6 & 3 \\
\hline Stomachs & $40^{v}$ & 4 & 12 & 0 & $34^{v}$ & 8 & $35^{v}$ & 3 & 4 \\
\hline Kidneys & $39^{v}$ & 1 & $24^{\mathrm{IV}}$ & 0 & $31^{v}$ & 2 & $33^{v}$ & 6 & 11 \\
\hline Spleen & $47^{v}$ & 1 & $29^{\mathrm{IV}}$ & 0 & $158^{\mathrm{VI}}$ & 0 & $32^{v}$ & 8 & 5 \\
\hline Tongues & $31^{v}$ & 1 & 13 & 0 & 10 & 0 & $25^{\mathrm{IV}}$ & 5 & 5 \\
\hline \multicolumn{10}{|c|}{$\begin{array}{l}\text { " } 90 \mathrm{~g} \text { is the prescribed portion size for lean meat according to the Food-based dietary guidelines for South Africans } \\
\text { (Schönfeldt, Pretorius, \& Hall, 2013) } \\
\text { "Values do not take bioavailability into account } \\
\text { " " Source of" as per the Foodstuffs, Cosmetics and Disinfectants act }(D O H, 2014)\end{array}$} \\
\hline \multicolumn{10}{|c|}{ v" "High in" as per the Foodstuffs, Cosmetics and Disinfectants act $(D O H, 2014)$} \\
\hline
\end{tabular}




\section{Reducing food waste with the consumption of lamb and mutton organs}

Apart from having the potential to be promoted as affordable animal source foods with a high yield percentage and low cooking losses, the consumption of organ meats is also directly related to minimising overall food waste (Jayathilakan, Sultana, Radhakrishna, \& Bawa, 2012). "Offal", which is the name given to organ meats, can be translated in plain English as "leftovers". Organ meats are the edible by-products left over after a carcass has been slaughtered and into desired cuts for formal sale. The 2011 Foresight report published in the UK with the title "Future of food and farming: Challenges and choices for global sustainability", explores the challenges relating to balancing sustainable food systems and public health matters (Government Office for Science, 2011). The report identified the need to change consumption patterns and improve the use of food by-products to reduce food waste. Organ meats have a high percentage edible portion fraction, containing no bone and minimal cartilage. In the light of the current nutrition situation in South Africa together with the global fight against food waste this nutrient dense food product such as offal meat products that often goes to waste, needs to be developed as food commodities.

\section{Conclusion and Recommendations}

The results of this study found that lamb and mutton organ meats are nutrient dense animal source foods. In the correct portion size, each organ proved to be either a "source of" high in or an "excellent source of" at least three different nutrients (included in the study). In the case of protein, zinc and iron, three nutrients of concern in South Africa, all lamb and mutton organ meats were at least a source of two out of these three nutrients with lamb and mutton spleens and lamb and mutton lungs being excellent sources of protein.

This data will also aid in the compilation of more accurate quantitative portion size recommendations. For example a single mutton kidney yields on average $29.1 \mathrm{~g}$ of edible portion (Table 2) which would mean that individuals would have to consume about 3 kidneys to adhere to the recommended portion size of $90 \mathrm{~g}$ cooked.

Considering the high levels of crude protein found in cooked lamb and mutton offal cuts, it is recommended that further research should be carried out on the amino acid profiles of these cuts to determine their protein quality. Furthermore, fatty acids also need to be 
determined in order that the contribution of offal to essential fatty acid intake can be determined. Knowledge of haem iron will also give an indication of the bioavailability of iron in offal.

\section{Funding}

This project was funded by the Research Technology Fund of the National Research Foundation and Red Meat Research and Development of South Africa (RTF14012862501) (RTF150327116211).

\section{Acknowledgements}

The authors would like to thank the staff at the Department of Consumer Sciences as well as the Nutrilab at the University of Pretoria for all their assistance with the preparation and analysis of the samples.

\section{References}

AOAC International. (2000). Official method of analysis 934.01; 942.05; 954.02;968.06 (17th Edition) Volume I. Association of Official Analytical Chemists, Inc., Maryland, USA.

Brown, A. (2010). Understanding Food: Principles and Preparation (4th ed.). Wadsworth Publishing .

Buttriss, J., \& Riley, H. (2013). Sustainable diets: Harnessing the nutrition agenda. Food Chemistry, 140(3), 402-407.

DOH. (2014). Draft Guidelines to the draft Regulations Relating to the Labeling and Advertising of Foods (R429 of 29 May 2014). Government Gazette, No. 10203.

Duvenage, S. S., Schönfeldt, H. C., \& Vermeulen, H. (2011). Perceptions and behaviour of lower socio-economic subgroups in the Limpopo province of South African towards Red Meat. Pretoria: Report to RMRD SA.

FAO. (2014, November). ICN Second International Conference on Nutrition: better nutrition, better lives. Retrieved December 8, 2014, from Food and Agriculture Organization of the United Nations: http://www.fao.org/about/meetings/icn2/documents/en/ 
Giron, H, C., (1973). Perkin Elmer Atomic Spectrophotometer. Atomic Absorption Newsletter $12: 28$

Government Office for Science. (2011). The future of food and farming: Challanges and choices for global sustainability. London: The Government Office for Science.

Jayathilakan, K., Sultana, K., Radhakrishna, K., \& Bawa, A. S. (2012). Utilization of byproducts and waste materials from meat, poultry and fish processing industries: a review. Journal of Food Science and Technology, 49(3), 278-293.

Khalafalla, F. A., Abdel Atty, N. S., Abd-El-Wahab, M. A., Omima, I. A., \& Abo-Elsoud, R. B. (2015). Assessment if heavy metal residues in retail meat and offals. Journal of American Science, 11(5), 50-54.

Labadarios, D., Maunder, E., Steyn, N., Maclntyre, U., Swart, R., Gericke, G., \& Dannhauser, A. (2003). National food consumption survey in children aged 1-9 years: South Africa 1999. South African Journal of Clinical Nutrition, 56, 106-109.

Labadarios, D., Steyn, N.P., Maunder, E., Maclntyre, U., Gericke, G., Swart, R., Huskisson, J., Dannhauser, A., Vorster, H.H., Nesmvuni, A. E. and Nel, J.H. (2005). The National Food Consumption Survey (NFCS): South Africa, 1999. Public Health Nutrition, 8(5), 533-543.

McAfee, A. J., McSorley, E. M., Cuskelly, G. J., Moss, B. W., Walleace, J. M., Bonham, M. P., \& Fearon, A. M. (2010). Red meat consumption: An overview of the risks and benefits. Meat Science, 84, 1-13.

Purchas, R. W., \& Wilkinson, B. H. (2013). The concentration of selected nutrients in New Zealand beef and lamb cuts and offal items. Institute of Food, Nutrition and Human Health. Palmerston North: Massey University.

Sainsbury, J., Schönfeldt, H. C., \& Van Heerden, S. M. (2011). The nutrient composition of South African mutton. Journal of Food Composition and Analysis, 24(4-5), 720-726.

Schönfeldt, H. C., Pretorius, B., \& Hall, N. (2013). Fish, Chicken, Lean meat or eggs can be eaten daily. South African Journal of Nutrition, Accepted.

Shisana, O., Labadarios, D., Rehle, D., Simbayi, L., Zuma, K., Dhansay, A., Reddy, P., Parker, W., Hoosain, E., Naidoo, E., Hongoro, C., Mchiza, Z., Steyn, N.P., Dwane, N., Makoae, M., Maluleke, T., Ramlagan, S., Zungu, N., Evans, M.G., Jacobs, L., Faber, M.. (2014). South African National Health and Nutrition Examination Survey. Cape Town: HSRC Press.

South African Meat Industry Company. (2016, July 24). Classification of Red Meat in South Africa. Retrieved from SAMIC: http://www.samic.co.za/downloads/Redmeat.pdf 
Tshabalala, P. A., Strydom, P. E., Webb, E. C., \& De Kock, H. L. (2003). Meat quality of designated South African indiginous got and sheep breeds. Meat Science, 65(1), 563570.

Van Heerden, S. M., \& Morey, L. (2014). Nutrient content of South African C2 beef offal. Journal of Food Measurment and Characterization, 8(3), 249-258.

Vermeulen, H., Schönfeldt, H. C., \& Pretorius, B. (2014). A survey to investigate SA consumers' perception towards red meat. Pretoria: Report to RMRD SA.

WEF. (2015, January 21). 5 reasons to be optimistic about Africa. Retrieved from World Economic Forum: https://agenda.weforum.org/2015/01/5-reasons-to-be-optimisticabout-africa/

Windows Genstats. (2000). Oxford, UK: VSN International LTD. 


\section{Supplement 1: Methods of analysis references}

\begin{tabular}{|c|c|}
\hline \multicolumn{2}{|r|}{ General Analyses } \\
\hline Moisture Determination & $\begin{array}{l}\text { AOAC, 2000. Official method of analysis } 934.01\left(17^{\text {th }} \text { Edition) Volume I. }\right. \\
\text { Association of Official Analytical Chemists, Inc., Maryland, USA }\end{array}$ \\
\hline $\begin{array}{l}\text { Dry Matter Ashing (inorganic } \\
\text { fraction) }\end{array}$ & $\begin{array}{l}\text { AOAC, 2000. Official method of analysis } 932.05\left(17^{\text {th }} \text { Edition) Volume I. }\right. \\
\text { Association of Official Analytical Chemists, Inc., Maryland, USA }\end{array}$ \\
\hline Crude protein (CP) & $\begin{array}{l}\text { AOAC, 2000. Official method of analysis } 968.06\left(17^{\text {th }} \text { Edition) Volume I. }\right. \\
\text { Association of Official Analytical Chemists, Inc., Maryland, USA }\end{array}$ \\
\hline $\begin{array}{l}\text { Ether Extraction (EE) for crude } \\
\text { fat }\end{array}$ & $\begin{array}{l}\text { AOAC, 2000. Official method of analysis } 920.39\left(17^{\text {th }} \text { Edition) Volume I. }\right. \\
\text { Association of Official Analytical Chemists, Inc., Maryland, USA }\end{array}$ \\
\hline \multicolumn{2}{|r|}{ Mineral Analysis } \\
\hline $\begin{array}{l}\text { Sample preparation (Ca, Mg, } \mathrm{Cu} \text {, } \\
\mathrm{Mn}, \mathrm{K}, \mathrm{Na}, \mathrm{Fe}, \mathrm{Zn})\end{array}$ & $\begin{array}{l}\text { AOAC, 2000. Official method of analysis } 935.13\left(17^{\text {th }} \text { Edition) Volume I. }\right. \\
\text { Association of Official Analytical Chemists, Inc., Maryland, USA }\end{array}$ \\
\hline Sample preparation (P) & $\begin{array}{l}\text { AOAC, 2000. Official method of analysis 968.08.D.b (17 }{ }^{\text {th }} \text { Edition) Volume I. } \\
\text { Association of Official Analytical Chemists, Inc., Maryland, USA }\end{array}$ \\
\hline $\begin{array}{l}\text { Mineral content (Ca, Mg, } \mathrm{Cu}, \mathrm{Mn} \text {, } \\
\mathrm{K}, \mathrm{Na}, \mathrm{Fe}, \mathrm{Zn})\end{array}$ & $\begin{array}{l}\text { Giron, H. C., 1973. Atomic Absorption Newsletter 12, 28. Perkin Elmer } \\
\text { Atomic Spectrophotometer }\end{array}$ \\
\hline Phosphorus (P) & $\begin{array}{l}\text { AOAC, 2000. Official method of analysis } 965.17\left(17^{\text {th }} \text { Edition) Volume I. }\right. \\
\text { Association of Official Analytical Chemists, Inc., Maryland, USA }\end{array}$ \\
\hline
\end{tabular}

